\title{
Noether Gauge Symmetries for Petrov Type D-Levi-Civita Space-Time in Spherical and Cylindrical Coordinates
}

\author{
Adil Jhangeer ${ }^{1}$ and Tayyaba $\mathrm{Naz}^{2}$ \\ ${ }^{1}$ Deanship of Educational Services, Preparatory Year Unit, Qassim University, P.O. Box 6595, Al-Qassim, Buraidah 51452, Saudi Arabia \\ ${ }^{2}$ Department of Sciences and Humanities, National University of Computer and Emerging Sciences, Lahore Campus, \\ Lahore 54700, Pakistan
}

Correspondence should be addressed to Adil Jhangeer; adil.jhangeer@gmail.com

Received 3 March 2016; Revised 11 May 2016; Accepted 12 May 2016

Academic Editor: Nilton O. Santos

Copyright ( 2016 A. Jhangeer and T. Naz. This is an open access article distributed under the Creative Commons Attribution License, which permits unrestricted use, distribution, and reproduction in any medium, provided the original work is properly cited.

Petrov Type D-Levi-Civita (DLC) space-time is considered in two different coordinates, that is, spherical and cylindrical. Noether gauge symmetries and their corresponding conserved quantities for respective metric with the restricted range of parameters and coordinates are discussed.

\section{Introduction}

Levi-Civita (LC) space-time is considered as the static, cylindrically symmetric vacuum solutions of Einstein's field equations. The vacuum space-time Petrov Type DLC is taken from Stephani et al. [1]. Ehlers and Kundt [2] studied this metric in spherical and cylindrical coordinates with the restricted range of parameters. Zinovyevich has been famous for classification of Einstein space, which is known as the Petrov classification. In 1954, the Weyl tensor which is related to Petrov classification is published for first time by A. Z. Petrov. In a Lorentzian manifold, the Weyl tensor having all possible algebraic symmetries is described by the Petrov classification in the field of theoretical physics and differential geometry. Space-time with different events has different types of Petrov. With respect to the field of general relativity, sometimes Petrov Types classification is called the gravitational field classification. So, this metric plays an important role in the field of general relativity; therefore, most of the different solutions for Levi-Civita space-time are investigated in recent years. The regions of Petrov Type D is related to the massive objects of the gravitational field. However, the characterization of mass and angular momentum of a gravitating object field occurs as the field of Petrov Type D. In some specific cases, a physical interpretation and close correspondence were found between the constant of symmetries and the Petrov Type. For instance, spherically static space-time is just like Petrov Type D. In general, spacetime is special type of Petrov Type I, but this space-time which is not geodesically complete is Petrov Type D space-time.

Symmetry plays an important role in understanding nature of the universe. Some of the stars are considered to have sphere-like symmetry. Cylindrical and plane symmetries are helpful to compute the gravitational waves. Since the field of general relativity and some other modified theories, like $f(R)[3-10]$ and $f(R, T)[11-15]$ theories of gravity and so forth, are assumed to be highly nonlinear, the solutions of their field equations can be found by their symmetries. Also in physics, symmetry has great significance to simplify the solutions of many problems. Thus, we can say a concept of symmetry is more helpful to describe the geometry of the space-time. In physics, the connection between the symmetries and conservation laws has both the fascinating and the fundamental significance.

In the 19th century, William Rowan Hamilton developed some alternative methods for calculating the conserved quantities. Another important approach, that is, Hamilton-Jacobi equation, is also helpful for finding the conserved quantities of DEs. But, here in this paper, Noether approach is used for finding the conserved quantities $[16,17]$ of the variational problems. This approach is frequently used in the areas of the theoretical physics. In this paper, we used Emmy Noether's 
approach to calculate the conserved quantities of the respective metric. A mathematician Emmy Noether (1812-1935) has been famous among the physicists. She has proved Noether's theorem in 1915 and it has been published in 1918. Noether's theorem states that there is a connection between the continuous symmetries of nature and the conserved quantities [16]. So, according to that theorem, we related the conservation energy to the time. In the field of modern theoretical physics and calculus of variations, Noether's theorem has great importance and it has also become a fundamental practical calculational tool. It helps us to investigate the (invariants) conserved quantities of a physical system. In physics, this theorem is considered as a powerful tool which states that every symmetry of a physical system provides a conserved quantity of the system [17]. So, in simple words, we can say that a one to one correspondence is found between the symmetries of nature and the laws of conservation of a physical system. When the symmetry is translation symmetric, then the theorem leads us to the linear conservation of momentum. If the physical system has rotational symmetry, then it gives the conservation of angular momentum and the symmetry in time provides the conservation of energy $[16,17]$. For the cosmological equation, we used systematic Noether's approach to obtain the exact solution of the system. In the field of theoretical physics and the calculus of variations, many applications of Noether's theorem have a vital role. The application of Noether's theorem helps the physicists to obtain the extremely powerful insights into any general theory of relativity and physics, by just observing the different transformations which has made the special form of laws involved invariant. In the field theory, Noether's theorem is developed for Lagrange-Euler's equation. However, from Noether's theorem, it is clear that generalized symmetries give the complete identification between the continuous symmetries and corresponding conserved quantities.

Thus, it would be interesting to study the Petrov Type D-Levi-Civita space-time from classification point of view in spherical and cylindrical coordinates. Ponce discussed the Levi-Civita space-time in multidimensional theories and also constructed the family of Levi-Civita-Kasner vacuum solutions in $(4+N)$ dimensions [18]. da Silva et al. [19] investigated two exact solutions of Einstein's field equations corresponding to a cylinder of dust with net zero angular momentum. He observed that the dust distribution is homogeneous in one of the cases, while, in the other, the angular velocity of dust particles is constant. The same authors [20] investigated the main properties of Levi-Civita solutions with the cosmological constant. Weinstein developed correspondence between Einstein and Levi-Civita [21]. Konkowski et al. [22] worked on the quantum singularity of Levi-Civita space-time. Miguelote et al. discussed the local and global properties of the Levi-Civita solutions coupled with an electromagnetic field [23].

Noether symmetries play an important role in recovering some lost conservation laws and the symmetry generators of space-time [24-26]. Sharif and Waheed [27] explored the energy contents of colliding plane waves by using the approximate Noether symmetry approach. It has been observed that there does not exist any nontrivial first-order symmetry generator for plane electromagnetic and gravitational waves. The same authors [28] investigated the energy contents of Bardeen model and stringy charged black hole type solutions using approximate Lie symmetries method. Shamir et al. [29] calculated $f(R)$ theory of gravity in the presence of gauge term through Noether symmetries approach. For this purpose, they considered Noether symmetry generators for spherically symmetric space-time and FriedmannRobertson-Walker universe. Capozziello et al. [5] established $f(R)$ gravity for spherically symmetric space-time using approximate Noether symmetries. In [30], a new class of Noether symmetries has been observed for spherical symmetry in $f(R)$ gravity. Using the Lie and Noether point operators, Paliathanasis et al. [31] discussed the modified $f(R)$ gravity models.

The pattern of paper is organized as follows: Section 1.1 is devoted to present the fundamental operators. Section 2 presents the Noether gauge operators and conserved quantities of the Petrov Type DLC in spherical and cylindrical coordinates by using Noether's approach. The last section of the paper contains the summary and conclusion.

1.1. Fundamental Operators. The method for calculating the Noether symmetries by using the Lagrangian is defined as follows $[32,33]$. We consider a vector field $X$ as

$$
X=\xi\left(s, x^{a}\right) \frac{\partial}{\partial s}+\eta^{b}\left(s, x^{a}\right) \frac{\partial}{\partial x^{b}} .
$$

Then $X$ is said to be the Noether point symmetry of the Lagrangian that satisfied this relation:

$$
X^{[1]}(L)+L D_{s}(\xi)=D_{s}(A),
$$

where $X^{[1]}$ is the first-order prolongation [34]. $A$ is said to be gauge terms and the total derivative operator $D$ is defined as

$$
D_{s}=\frac{\partial}{\partial s}+\dot{x}^{a} \frac{\partial}{\partial x^{a}} .
$$

The first integral of motion associated with $X$ is defined as

$$
I=\xi L+\left(\eta^{a}-\xi \dot{x}^{a}\right) \frac{\partial L}{\partial \dot{x}^{a}}-A .
$$

\section{Noether Gauge Operators and Conserved Quantities}

In this section, Noether gauge operators and conserved quantities of the Petrov Type DLC in spherical and cylindrical coordinates are computed by using the Noether approach.

\subsection{Petrov Type DLC in Spherical Coordinates}

2.1.1. Type 1. Levi-Civita space-time in spherical coordinates, $(t, r, \theta, \phi)$, is

$$
\begin{aligned}
d s^{2}= & r^{2}\left(d \theta^{2}+\sin ^{2} \theta d \phi^{2}\right)+\left(\frac{r}{r-b}\right) d r^{2} \\
& -\left(\frac{r-b}{r}\right) d t^{2}
\end{aligned}
$$


The coordinates and parameters are restricted as $t \in R, 0<$ $\theta<\pi, \phi \in[0,2 \pi)$, and $(0<b<r) \vee(b<0<r)$.

The Lagrangian for Levi-Civita space-time in spherical coordinates (5) is

$$
L=r^{2}\left(\dot{\theta}^{2}+\sin ^{2} \theta \dot{\phi}^{2}\right)+\left(\frac{r}{r-b}\right) \dot{r}^{2}-\left(\frac{r-b}{r}\right) \dot{t}^{2} .
$$

Now substituting (6) in (2) and after some manipulations, we get the following system of linear PDEs:

$$
\begin{aligned}
& \text { (i) } \xi_{\phi}=0 \text {, } \\
& \text { (ii) } \xi_{\theta}=0 \text {, } \\
& \text { (iii) } \xi_{r}=0 \text {, } \\
& \text { (iv) } \xi_{t}=0 \text {, } \\
& \text { (v) } A_{s}=0 \text {, } \\
& \text { (i) }-2\left(\frac{r-b}{r}\right) \eta_{s}^{1}=A_{t} \text {, } \\
& \text { (ii) }\left(\frac{2 r}{r-b}\right) \eta_{s}^{2}=A_{r} \text {, } \\
& \text { (iii) } 2 r^{2} \eta_{s}^{3}=A_{\theta} \text {, } \\
& \text { (i) } 2 r^{2} \sin ^{2} \theta \eta_{s}^{4}=A_{\phi} \text {, } \\
& \text { (ii) }\left(\frac{r-b}{r}\right) \eta_{r}^{1}-\left(\frac{r}{r-b}\right) \eta_{t}^{2}=0 \text {, } \\
& \text { (iii) }\left(\frac{r-b}{r}\right) \eta_{\theta}^{1}-r^{2} \eta_{t}^{3}=0 \text {, } \\
& \text { (i) }\left(\frac{r+b}{r}\right) \eta_{\phi}^{1}+r^{2} \sin ^{2} \theta \eta_{t}^{4}=0 \text {, } \\
& \text { (ii) }\left(\frac{1}{r-b}\right) \eta_{\theta}^{2}+r \eta_{r}^{3}=0 \text {, } \\
& \text { (iii) }\left(\frac{1}{r-b}\right) \eta_{\phi}^{2}+r \sin ^{2} \theta \eta_{r}^{4}=0 \text {, } \\
& \text { (i) } \eta_{\phi}^{3}+\sin ^{2} \theta \eta_{\theta}^{4}=0 \text {, } \\
& \text { (ii) } r \xi_{s}-2 \eta^{2}-2 r \eta_{\theta}^{3}=0 \text {, } \\
& \text { (iii) } \quad(r-b) \xi_{s}-\left(\frac{b}{r}\right) \eta^{2}-2(r-b) \eta_{t}^{1}=0, \\
& \text { (i) } r \xi_{s}+\left(\frac{b}{r-b}\right) \eta^{2}-2 r \eta_{r}^{2}=0 \text {, } \\
& \text { (ii) } r \xi_{s}-2 r \eta_{\phi}^{4}-2 \eta^{2}-2 r \cot \theta \eta^{3}=0 .
\end{aligned}
$$

For this case, the solution is

$$
\begin{aligned}
\xi & =c_{1}, \\
\eta^{1} & =c_{3}, \\
\eta^{i} & =0, \quad i=2,3,4, \\
A & =c_{2} .
\end{aligned}
$$

Thus, the approximate Noether operators are $X_{1}=\partial / \partial s, X_{2}=$ $\partial / \partial t$. The first integrals of motion associated with approximate Noether point symmetries $X_{1}$ and $X_{2}$ are

$$
\begin{aligned}
I_{1} & =\left(\frac{r-b}{r}\right) \dot{t}^{2}-\left(\frac{r}{r-b}\right) \dot{r}^{2}-r^{2} \dot{\theta}^{2}-r^{2} \dot{\phi}^{2} \sin ^{2} \theta-c_{2}, \\
I_{2} & =-2\left(\frac{r-b}{r}\right) \dot{t}-c_{2} .
\end{aligned}
$$

2.1.2. Type 2. The metric is given by the line element in the spherical coordinates $(t, r, \theta, \phi)$ :

$$
\begin{aligned}
d s^{2}= & r^{2}\left(d \theta^{2}-\sin ^{2} \theta d t^{2}\right)+\left(\frac{r}{r-b}\right) d r^{2} \\
& +\left(\frac{r-b}{r}\right) d \phi^{2} .
\end{aligned}
$$

The coordinates and parameters are restricted to $t \in R, 0<$ $\theta<\pi, \phi \in[0,2 \pi)$, and $(0<b<r) \vee(b<0<r)$.

The Lagrangian for (15) is defined as

$$
L=r^{2}\left(\dot{\theta}^{2}-\sin ^{2} \theta \dot{t}^{2}\right)+\left(\frac{r}{r-b}\right) \dot{r}^{2}+\left(\frac{r-b}{r}\right) \dot{\phi}^{2} .
$$

Lagrangian (16) with (2) yields

$$
\begin{aligned}
& \text { (i) } \xi_{\phi}=0 \text {, } \\
& \text { (ii) } \xi_{\theta}=0 \text {, } \\
& \text { (iii) } \xi_{r}=0 \text {, } \\
& \text { (iv) } \xi_{t}=0 \text {, } \\
& \text { (v) } A_{s}=0 \text {, } \\
& \text { (i) }-2 r^{2} \sin ^{2} \theta \eta_{s}^{1}=A_{t} \text {, } \\
& \text { (ii) }\left(\frac{2 r}{r-b}\right) \eta_{s}^{2}=A_{r} \text {, } \\
& \text { (iii) } 2 r^{2} \eta_{s}^{3}=A_{\theta} \text {, } \\
& \text { (i) } 2\left(\frac{r-b}{r}\right) \eta_{s}^{4}=A_{\phi} \text {, } \\
& \text { (ii) } r \sin ^{2} \theta \eta_{r}^{1}-\left(\frac{1}{r-b}\right) \eta_{t}^{2}=0 \text {, } \\
& \text { (iii) } \sin ^{2} \theta \eta_{\theta}^{1}-\eta_{t}^{3}=0 \text {, } \\
& \text { (i) } r^{2} \sin ^{2} \theta \eta_{\phi}^{1}-\left(\frac{r-b}{r}\right) \eta_{t}^{4}=0, \\
& \text { (ii) }\left(\frac{1}{r-b}\right) \eta_{\theta}^{2}+r \eta_{r}^{3}=0 \\
& \text { (iii) }\left(\frac{r}{r-b}\right) \eta_{\phi}^{2}+\eta_{r}^{4}-\left(\frac{b}{r}\right) \eta_{r}^{4}=0 \text {, }
\end{aligned}
$$



(i) $r^{2} \eta_{\phi}^{3}+\left(\frac{r-b}{r}\right) \eta_{\theta}^{4}=0$
(ii) $r \xi_{s}-2 \eta^{2}-2 r \eta_{\theta}^{3}=0$,
(iii) $r \xi_{s}+\left(\frac{b}{r-b}\right) \eta^{2}-2 r \eta_{r}^{2}=0$
(i) $r \xi_{s}-2 \eta^{2}-2 r \eta_{t}^{1}-2 r \cot \theta \eta^{3}=0$,
(ii) $(r-b) \xi_{s}-\left(\frac{b}{r}\right) \eta^{2}-2(r-b) \eta_{\phi}^{4}=0$.

For this case, the solution of the above equation will be

$$
\begin{aligned}
\xi & =c_{1}, \\
\eta^{4} & =c_{3}, \\
\eta^{i} & =0, \quad i=1,2,3, \\
A & =c_{2} .
\end{aligned}
$$

Thus, the approximate Noether operators will be $X_{1}=\partial / \partial s$, $X_{2}=\partial / \partial \phi$. Corresponding first integrals of motion for approximate Noether point symmetries are

$$
\begin{aligned}
& I_{1}=r^{2} \sin ^{2} \theta \dot{t}^{2}-\left(\frac{r}{r-b}\right) \dot{r}^{2}-r^{2} \dot{\theta}^{2}-\left(\frac{r-b}{r}\right) \dot{\phi}-c_{2}, \\
& I_{2}=2\left(\frac{r-b}{r}\right) \dot{\phi}-c_{2} .
\end{aligned}
$$

\subsection{Petrov Type DLC in Cylindrical Coordinates}

2.2.1. Type 1. In cylindrical coordinates, Levi-Civita spacetime is defined as

$$
\begin{aligned}
d s^{2}= & z^{2}\left(d r^{2}+\sinh ^{2} r d \phi^{2}\right)+\left(\frac{z}{b-z}\right) d z^{2} \\
& -\left(\frac{b-z}{z}\right) d t^{2}
\end{aligned}
$$

with the restrictions on coordinates and parameters as $t \in R$, $0<r, \phi \in[0,2 \pi)$, and $(0<z<b)$.

The Lagrangian for (25) is

$$
L=z^{2}\left(\dot{r}^{2}+\sinh ^{2} r \dot{\phi}^{2}\right)+\left(\frac{z}{b-z}\right) \dot{z}^{2}-\left(\frac{z-b}{z}\right) \dot{t}^{2} .
$$

Equation (26) with the help of (2) gives
(i) $\xi_{\phi}=0$,
(ii) $\xi_{z}=0$,
(iii) $\xi_{r}=0$,
(iv) $\xi_{t}=0$,
(v) $A_{s}=0$,

(i) $2\left(\frac{z-b}{z}\right) \eta_{s}^{1}=A_{t}$,

(ii) $2 z^{2} \eta_{s}^{2}=A_{r}$,

(iii) $\left(\frac{2 z}{b-z}\right) \eta_{s}^{3}=A_{z}$,

(i) $2 z^{2} \sinh ^{2} r \eta_{s}^{4}=A_{\phi}$,

(ii) $\left(\frac{z-b}{z}\right) \eta_{r}^{1}+z^{2} \eta_{t}^{2}=0$,

(iii) $\left(\frac{z-b}{z}\right) \eta_{z}^{1}+\left(\frac{z}{b-z}\right) \eta_{t}^{3}=0$,

(i) $\left(\frac{z-b}{z}\right) \eta_{\phi}^{1}+z^{2} \sinh ^{2} r \eta_{t}^{4}=0$,

(ii) $z \eta_{z}^{2}+\left(\frac{1}{b-z}\right) \eta_{r}^{3}=0$

(iii) $\eta_{\phi}^{2}+\sinh ^{2} r \eta_{r}^{4}=0$,

(i) $\left(\frac{1}{b-z}\right) \eta_{\phi}^{3}+z \sinh ^{2} r \eta_{z}^{4}=0$,

(ii) $z \xi_{s}-2 \eta^{3}-2 z \eta_{r}^{2}=0$,

(i) $(z-b) \xi_{s}-\left(\frac{b}{z}\right) \eta^{3}-2(z-b) \eta_{t}^{1}=0$,

(ii) $z \xi_{s}-\left(\frac{b}{b-z}\right) \eta^{3}-2 z \eta_{z}^{3}=0$,

$$
z \xi_{s}-2 z \eta_{\phi}^{4}-2 \eta^{3}-2 z \operatorname{coth} r \eta^{2}=0 .
$$

In this case, the solution is

$$
\begin{aligned}
\xi & =c_{1}, \\
\eta^{1} & =c_{3}, \\
\eta^{2} & =-c_{4} \cos \phi+c_{5} \sin \phi, \\
\eta^{3} & =0, \\
\eta^{4} & =\frac{1}{\sinh r}\left[c_{4} \sin \phi+c_{5} \cos \phi \cosh r+c_{6} \sinh r\right], \\
A & =c_{2} .
\end{aligned}
$$

We obtained these five Noether operators:

$$
\begin{aligned}
& X_{1}=\frac{\partial}{\partial s}, \\
& X_{2}=\frac{\partial}{\partial t}, \\
& X_{3}=-\cos \phi \frac{\partial}{\partial r}+\frac{\sin \phi}{\sinh r} \frac{\partial}{\partial \phi}, \\
& X_{4}=\sin \phi \frac{\partial}{\partial r}+\frac{\cos \phi \cosh r}{\sinh r} \frac{\partial}{\partial \phi}, \\
& X_{5}=\frac{\partial}{\partial \phi} .
\end{aligned}
$$


The above solution gave five symmetries, while three symmetries were the same as the symmetries in spherical coordinates.

The first integrals of motion associated with approximate Noether point symmetries $X_{1}, X_{2}, X_{3}, X_{4}$, and $X_{5}$ by using (4) as follows:

$$
\begin{aligned}
I_{1}= & 2 z^{2} \dot{r}-c_{2}, \\
I_{2}= & 2 z^{2} \sin ^{2} h r \dot{\phi}-c_{2}, \\
I_{3}= & \left(\frac{b-z}{z}\right) \dot{t}^{2}-z^{2} \dot{r}^{2}-\left(\frac{z}{b-z}\right) \dot{z}^{2}-z^{2} \dot{\phi}^{2} \sin ^{2} h r \\
& -c_{2}, \\
I_{4}= & -2 z^{2} \cos \phi \dot{r}+2 z^{2} \sin \phi \sinh r \dot{\phi}-c_{2}, \\
I_{5}= & 2 z^{2} \sin \phi \dot{r}+2 z^{2} \cos \phi \cosh r \sinh r \dot{\phi}-c_{2} .
\end{aligned}
$$

2.2.2. Type 2. The Levi-Civita metric in the cylindrical coordinates $(t, r, z, \phi)$ is given as

$$
d s^{2}=z^{2}\left(d r^{2}+r^{2} d \phi^{2}\right)+z d z^{2}-\frac{1}{z} d t^{2}
$$

where the restriction of coordinates is $t \in R, 0<r, \phi \in[0$, $2 \pi$ ), and $0<z$.

The Lagrangian for (37) is defined as

$$
L=z^{2}\left(\dot{r}^{2}+r^{2} \dot{\phi}^{2}\right)+z \dot{z}^{2}-\frac{1}{z} \dot{t}^{2}
$$

Lagrangian (38) with (2) gives the following system of determining equations:
(i) $\xi_{\phi}=0$,
(ii) $\xi_{z}=0$,
(iii) $\xi_{r}=0$,
(iv) $\xi_{t}=0$,
(v) $A_{s}=0$,
(i) $-\left(\frac{2}{z}\right) \eta_{s}^{1}=A_{t}$,
(ii) $2 z^{2} \eta_{s}^{2}=A_{r}$,
(iii) $2 z \eta_{s}^{3}=A_{z}$,
(i) $2 r^{2} z^{2} \eta_{s}^{4}=A_{\phi}$,
(ii) $\left(\frac{1}{z}\right) \eta_{r}^{1}-z^{2} \eta_{t}^{2}=0$,
(iii) $\left(\frac{1}{z}\right) \eta_{z}^{1}-z \eta_{t}^{3}=0$,
(i) $\left(\frac{1}{z}\right) \eta_{\phi}^{1}-z^{2} r^{2} \eta_{t}^{4}=0$,
(ii) $z \eta_{z}^{2}+\eta_{r}^{3}=0$,
(iii) $\eta_{\phi}^{2}+r^{2} \eta_{r}^{4}=0$,
(i) $\eta_{\phi}^{3}+z r^{2} \eta_{z}^{4}=0$,
(ii) $z \xi_{s}-2 \eta^{3}-2 \eta_{r}^{2}=0$,
(iii) $\xi_{s}-2 \eta_{t}^{1}+\left(\frac{1}{z}\right) \eta^{3}=0$,
(i) $z \xi_{s}-\eta^{3}-2 z \eta_{z}^{3}=0$,
(ii) $z r \xi_{s}-2 z \eta^{2}-2 r \eta^{3}-2 r z \eta_{\phi}^{4}=0$.

For this case, the above equations yield the solution

$$
\begin{aligned}
\xi & =c_{1} s+c_{2}, \\
\eta^{1} & =\frac{2}{3} c_{1} t+c_{4}, \\
\eta^{2} & =\frac{1}{6} r c_{1}-c_{5} \cos \phi+c_{6} \sin \phi, \\
\eta^{3} & =\frac{1}{3} z c_{1}, \\
\eta^{4} & =\frac{1}{r}\left[c_{7} r+c_{5} \sin \phi+c_{6} \cos \phi\right], \\
A & =c_{3} .
\end{aligned}
$$

The Noether operators are

$$
\begin{aligned}
& X_{1}=s \frac{\partial}{\partial s}+\frac{2}{3} t \frac{\partial}{\partial t}+\frac{1}{6} r \frac{\partial}{\partial r}+\frac{1}{3} z \frac{\partial}{\partial z} \\
& X_{2}=\frac{\partial}{\partial s}, \\
& X_{3}=\frac{\partial}{\partial t}, \\
& X_{4}=-\cos \phi \frac{\partial}{\partial r}+\frac{1}{r} \sin \phi \frac{\partial}{\partial \phi}, \\
& X_{5}=\sin \phi \frac{\partial}{\partial r}+\frac{1}{r} \cos \phi \frac{\partial}{\partial \phi}, \\
& X_{6}=\frac{\partial}{\partial \phi} .
\end{aligned}
$$

Then, the first integrals of motion corresponding to the above Noether operators by using (4) are

$$
\begin{aligned}
& I_{1}=-\frac{2}{z} \dot{t}-c_{3}, \\
& I_{2}=2 z^{2} r^{2} \dot{\phi}-c_{3},
\end{aligned}
$$




$$
\begin{aligned}
I_{3}= & \frac{1}{z} \dot{t}^{2}-z^{2} \dot{r}^{2}-z \dot{z}^{2}-2 z^{2} r^{2} \dot{\phi}^{2}-\frac{4 t}{3 z} \dot{t}+\frac{r z^{2}}{3} \dot{r} \\
& +\frac{2 z^{2}}{3} \dot{z}-c_{3}, \\
I_{4}= & \frac{1}{z} \dot{t}^{2}-z^{2} \dot{r}^{2}-z \dot{z}^{2}-2 z^{2} r^{2} \dot{\phi}^{2}-c_{3}, \\
I_{5}= & -2 z^{2} \cos \phi \dot{r}+2 r z^{2} \sin \phi \dot{\phi}-c_{3}, \\
I_{6}= & 2 z^{2} \sin \phi \dot{r}+2 r z^{2} \cos \phi \dot{\phi}-c_{3} .
\end{aligned}
$$

2.2.3. Type 3. The metric is given by the line element in cylindrical coordinates:

$$
\begin{aligned}
d s^{2}= & z^{2}\left(d r^{2}-\sinh ^{2} r d t^{2}\right)+\left(\frac{z}{b-z}\right) d z^{2} \\
& +\left(\frac{b-z}{z}\right) d \phi^{2}
\end{aligned}
$$

and the restrictions are $t \in R, \phi \in[0,2 \pi),(0<z<b)$, and $0<r$.

The Lagrangian for (48) is defined as

$$
L=z^{2}\left(\dot{r}^{2}-\sinh ^{2} r \dot{t}^{2}\right)+\left(\frac{z}{b-z}\right) \dot{z}^{2}+\left(\frac{z-b}{z}\right) \dot{\phi}^{2} .
$$

Now, for (49) by using (2), we get the following system of linear PDEs:
(i) $\xi_{\phi}=0$,
(ii) $\xi_{z}=0$,
(iii) $\xi_{r}=0$,
(iv) $\xi_{t}=0$,
(v) $A_{s}=0$,
(i) $-2 z^{2} \sinh ^{2} r \eta_{s}^{1}=A_{t}$,
(ii) $2 z^{2} \eta_{s}^{2}=A_{r}$,
(iii) $\left(\frac{2 z}{b-z}\right) \eta_{s}^{3}=A_{z}$,
(i) $-2\left(\frac{z-b}{z}\right) \eta_{s}^{4}=A_{\phi}$,
(ii) $\sinh ^{2} r \eta_{r}^{1}-\eta_{t}^{2}=0$,
(iii) $z \sinh ^{2} r \eta_{z}^{1}-\left(\frac{1}{b-z}\right) \eta_{t}^{3}=0$
(i) $z^{2} \sinh ^{2} r \eta_{\phi}^{1}+\left(\frac{z-b}{z}\right) \eta_{t}^{4}=0$,
(ii) $z \eta_{z}^{2}+\left(\frac{1}{b-z}\right) \eta_{r}^{3}=0$,
(iii) $z^{2} \eta_{\phi}^{2}-\eta_{r}^{4}+\left(\frac{b}{z}\right) \eta_{r}^{4}=0$,
(i) $\left(\frac{z}{b-z}\right) \eta_{\phi}^{3}-\left(\frac{z-b}{z}\right) \eta_{z}^{4}=0$,
(ii) $z \xi_{s}-2 \eta^{3}-2 z \eta_{r}^{2}=0$,
(iii) $z \xi_{s}-2 z \eta_{t}^{1}-2 \operatorname{coth} r \eta^{2}-2 \eta^{3}=0$,
(i) $z \xi_{s}-\left(\frac{b}{b-z}\right) \eta^{3}-2 z \eta_{z}^{3}=0$,
(ii) $(z-b) \xi_{s}-\left(\frac{b}{z}\right) \eta^{3}-2(z-b) \eta_{\phi}^{4}=0$.

For this case, (50)-(55) yield the solution

$$
\begin{gathered}
\xi=c_{1}, \\
\eta^{1}=c_{3}, \\
\eta^{2}=0, \\
\eta^{3}=0, \\
\eta^{4}=c_{4}, \\
A=c_{2} .
\end{gathered}
$$

The Noether operators are

$$
\begin{aligned}
& X_{1}=\frac{\partial}{\partial s} \\
& X_{2}=\frac{\partial}{\partial t}, \\
& X_{3}=\frac{\partial}{\partial \phi}
\end{aligned}
$$

And the first integrals of motion for these approximate Noether operators by using (4) are

$$
\begin{aligned}
I_{1}= & -z^{2} \sinh ^{2} r \dot{t}^{2}-z^{2} \dot{r}^{2}-\left(\frac{z}{b-z}\right) \dot{z}^{2}-\left(\frac{b-z}{z}\right) \dot{\phi}^{2} \\
& -c_{2}, \\
I_{2}= & -2 z^{2} \sinh ^{2} r \dot{t}-c_{2}, \\
I_{3}= & 2\left(\frac{b-z}{z}\right) \dot{\phi}-c_{2} .
\end{aligned}
$$

2.2.4. Type 4. The metric in the cylindrical coordinates is defined as

$$
d s^{2}=z^{2}\left(d r^{2}-r^{2} d t^{2}\right)+z d z^{2}+\frac{1}{z} d \phi^{2} .
$$


The coordinates and parameters are restricted to $t \in R, \phi \in$ $[0,2 \pi), 0<z$, and $0<r$.

The Lagrangian for Levi-Civita space-time in (59) is defined as

$$
L=z^{2}\left(\dot{r}^{2}-r^{2} \dot{t}^{2}\right)+z \dot{z}^{2}+\frac{1}{z} \dot{\phi}^{2} .
$$

Now substituting (60) in (2), we get the following system of linear PDEs:

$$
\begin{aligned}
& \text { (i) } \xi_{\phi}=0 \text {, } \\
& \text { (ii) } \xi_{z}=0 \text {, } \\
& \text { (iii) } \xi_{r}=0 \text {, } \\
& \text { (iv) } \xi_{t}=0 \text {, } \\
& \text { (v) } A_{s}=0 \text {, } \\
& \text { (i) }-2 r^{2} z^{2} \eta_{s}^{1}=A_{t} \text {, } \\
& \text { (ii) } 2 z^{2} \eta_{s}^{2}=A_{r} \text {, } \\
& \text { (iii) } 2 z \eta_{s}^{3}=A_{z} \text {, } \\
& \text { (i) }\left(\frac{2}{z}\right) \eta_{s}^{4}=A_{\phi} \text {, } \\
& \text { (ii) } r^{2} \eta_{r}^{1}-\eta_{t}^{2}=0 \text {, } \\
& \text { (iii) } r^{2} z \eta_{z}^{1}-\eta_{t}^{3}=0 \text {, } \\
& \text { (i) } r^{2} z^{2} \eta_{\phi}^{1}-\left(\frac{1}{z}\right) \eta_{t}^{4}=0 \text {, } \\
& \text { (ii) } z \eta_{z}^{2}+\eta_{r}^{3}=0 \text {, } \\
& \text { (iii) } z^{2} \eta_{\phi}^{2}+\left(\frac{1}{z}\right) \eta_{r}^{4}=0 \text {, } \\
& \text { (i) } z \eta_{\phi}^{3}+\left(\frac{1}{z}\right) \eta_{z}^{4}=0 \text {, } \\
& \text { (ii) } z \xi_{s}-2 \eta^{3}-2 z \eta_{r}^{2}=0 \text {, } \\
& \text { (iii) } r z \xi_{s}-2 r z \eta_{t}^{1}-2 z \eta^{2}-2 r \eta^{3}=0 \text {, } \\
& \text { (i) } z \xi_{s}-\eta^{3}-2 z \eta_{z}^{3}=0 \text {, } \\
& \text { (ii) } \xi_{s}-2 \eta_{\phi}^{4}+\left(\frac{1}{z}\right) \eta^{3}=0 \text {. }
\end{aligned}
$$

After some manipulations, we get the solution

$$
\begin{aligned}
\xi & =c_{1} s+c_{2}, \\
\eta^{1} & =0, \\
\eta^{2} & =\frac{1}{6} r c_{1}, \\
\eta^{3} & =\frac{1}{3} z c_{1}, \\
\eta^{4} & =\frac{2}{3} c_{1} \phi, \\
A & =c_{3} .
\end{aligned}
$$

The Noether operators obtained are

$$
\begin{aligned}
& X_{1}=s \frac{\partial}{\partial s}+\frac{1}{6} r \frac{\partial}{\partial r}+\frac{1}{3} z \frac{\partial}{\partial z}+\frac{2}{3} \phi \frac{\partial}{\partial \phi}, \\
& X_{2}=\frac{\partial}{\partial s} .
\end{aligned}
$$

Then, the first integrals of motion associated with Noether operators $X_{1}$ and $X_{2}$ by using (4) are

$$
\begin{aligned}
I_{1}= & s r^{2} z^{2} \dot{t}^{2}-s z^{2} \dot{r}^{2}-s z \dot{z}^{2}-\frac{s}{z} \dot{\phi}^{2}+\frac{r z^{2}}{3} \dot{r}+\frac{2 z^{2}}{3} \dot{z} \\
& +\frac{4 z}{3} \phi \dot{\phi}-c_{3}, \\
I_{2}= & r^{2} z^{2} \dot{t}^{2}-z^{2} \dot{r}^{2}-z \dot{z}^{2}-\frac{1}{z} \dot{\phi}^{2}-c_{3} .
\end{aligned}
$$

\section{Conclusion}

In this paper, we computed the Noether symmetries of Petrov Type DLC space-time in different coordinates in the presence of gauge term. For both spherical and cylindrical coordinates, we got an overdetermined system of nineteen linear partial differential equations. We found two Noether generators for first solution in the spherical coordinates, that is, $\partial / \partial s$ and $\partial / \partial t$, and for second solution we also got two symmetries. One symmetry $\partial / \partial s$ obtained in first solution is common and the additional symmetry is $\partial / \partial \phi$. Similarly, we investigated the Noether symmetries of all solutions in cylindrical coordinates. Further, corresponding energy contents are also discussed for each generator.

\section{Competing Interests}

The authors declare that there are no competing interests regarding the publication of this paper.

\section{References}

[1] H. Stephani, D. Kramer, M. MacCallum, C. Hoenselaers, and E. Herlt, Exact Solutions of Einstein's Field Equations, Cambridge Monographs on Mathematical Physics, Cambridge University Press, 2nd edition, 2003.

[2] J. Ehlers and W. Kundt, "Exact solutions of the gravitational field equations," in Gravitation: An Introduction to Current Research, pp. 49-101, John Wiley \& Sons, New York, NY, USA, 1962.

[3] H. A. Buchdahl, "Non-linear Lagrangians and cosmological theory," Monthly Notices of the Royal Astronomical Society, vol. 150, no. 1, pp. 1-8, 1970.

[4] T. Multamäki and I. Vilja, "Spherically symmetric solutions of modified field equations in $f(R)$ theories of gravity," Physical Review D, vol. 74, no. 6, Article ID 064022, 5 pages, 2006.

[5] S. Capozziello, A. Stabile, and A. Troisi, "Spherically symmetric solutions in $\mathrm{f}(\mathrm{R})$ gravity via the Noether symmetry approach," Classical and Quantum Gravity, vol. 24, no. 8, pp. 2153-2166, 2007.

[6] L. Hollenstein and F. S. N. Lobo, "Exact solutions of $f(R)$ gravity coupled to nonlinear electrodynamics," Physical Review D, vol. 78, no. 12, Article ID 124007, 2008. 
[7] A. Azadi, D. Momeni, and M. Nouri-Zonoz, "Cylindrical solutions in metric $\mathrm{f}(\mathrm{R})$ gravity," Physics Letters B, vol. 670, no. 3, pp. 210-214, 2008.

[8] M. Sharif and M. F. Shamir, "plane symmetric solutions in $f(R)$ gravity," Modern Physics Letters A, vol. 25, no. 15, pp. 1281-1288, 2010.

[9] M. Sharif and M. F. Shamir, "Exact solutions of Bianchi-type I and V spacetimes in the $f(R)$ theory of gravity," Classical and Quantum Gravity, vol. 26, no. 23, Article ID 235020, 2009.

[10] M. F. Shamir, "Some Bianchi type cosmological models in $f(R)$ gravity," Astrophysics and Space Science, vol. 330, no. 1, pp. 183189, 2010.

[11] T. Harko, F. S. N. Lobo, S. Nojiri, and S. D. Odintsov, “ $f(R, T)$ gravity," Physical Review D, vol. 84, no. 2, Article ID 024020, 11 pages, 2011.

[12] K. S. Adhav, "LRS Bianchi type-I cosmological model in $f(R, T)$ theory of gravity," Astrophysics and Space Science, vol. 339, no. 2, pp. 365-369, 2012.

[13] M. Sharif and M. Zubair, "Thermodynamics in $f(R, T)$ theory of gravity," Journal of Cosmology and Astroparticle Physics, vol. 2012, no. 3, article 028, 2012.

[14] M. J. S. Houndjo, "Reconstruction of $f(R, T)$ gravity describing matter dominated and accelerated phases," International Journal of Modern Physics D, vol. 21, Article ID 1250003, 14 pages, 2012.

[15] M. F. Shamir, A. Jhangeer, and A. A. Bhatti, "Exact solutions of Bianchi types $I$ and $V$ models in $f(R, T)$ gravity," https://arxiv .org/abs/1207.0708.

[16] N. Byers, "E. Noether's discovery of the deep connection between symmetries and conservation laws," in Israel Mathematical Conference Proceedings, vol. 12, pp. 67-82, 1999.

[17] E. Noether, "Invariante variationsprobleme," Nachrichten von der Königlichen Gesellschaft der Wissenschaften zu Göttingen (Math-Phys. Kl), vol. 2, pp. 235-257, 1918.

[18] J. Ponce de Leon, "Levi-Civita spacetimes in multidimensional theories," Modern Physics Letters A, vol. 24, no. 21, pp. 1659-1667, 2009.

[19] M. F. da Silva, L. Herrera, F. M. Paiva, and N. O. Santos, "The Levi-Civita space-time," Journal of Mathematical Physics, vol. 36, no. 7, pp. 3625-3631, 1995.

[20] M. F. da Silva, A. Wang, F. M. Paiva, and N. O. Santos, "LeviCivit solutions with a cosmological constant," Physical Review. D, vol. 61, no. 4, Article ID 044003, 2000.

[21] G. Weinstein, "Einstein the stubborn: correspondence between Einstein and Levi-Civita," https://arxiv.org/abs/1202.4305.

[22] D. A. Konkowski, T. M. Helliwell, and C. Wieland, "Quantum singularity of Levi-Civita spacetimes," Classical and Quantum Gravity, vol. 21, no. 1, pp. 265-272, 2004.

[23] A. Y. Miguelote, M. F. A. da Silva, A. Z. Wang, and N. O. Santos, "Levi-Civita solutions coupled with electromagnetic fields," Classical and Quantum Gravity, vol. 18, no. 21, pp. 45694588, 2001.

[24] T. Feroze, F. M. Mahomed, and A. Qadir, "The connection between isometries and symmetries of geodesic equations of the underlying spaces," Nonlinear Dynamics, vol. 45, no. 1-2, pp. 6574, 2006.

[25] I. Hussain, F. M. Mahomed, and A. Qadir, "Second-order approximate symmetries of the geodesic equations for the Reissner-Nordström metric and re-scaling of energy of a test particle," SIGMA, vol. 3, article 115, 2007.

[26] A. H. Kara, F. M. Mahomed, and A. Qadir, "Approximate symmetries and conservation laws of the geodesic equations for the Schwarzschild metric," Nonlinear Dynamics, vol. 51, no. 1-2, pp. 183-188, 2008.

[27] M. Sharif and S. Waheed, "Energy of the Bardeen model using an approximate symmetry method," Physica Scripta, vol. 83, no. 1, Article ID 015014, 2011.

[28] M. Sharif and S. Waheed, "Re-scaling of energy in stringy charged black hole solutions using approximate symmetries," Canadian Journal of Physics, vol. 88, no. 11, pp. 833-839, 2010.

[29] M. F. Shamir, A. Jhangeer, and A. A. Bhatti, "Conserved quantities in $f(R)$ gravity via noether symmetry," Chinese Physics Letters, vol. 29, no. 8, Article ID 080402, 2012.

[30] S. Capozziello, N. Frusciante, and D. Vernieri, "New spherically symmetric solutions in $\mathrm{f}(R)$-gravity by Noether symmetries," General Relativity and Gravitation, vol. 44, no. 8, pp. 1881-1891, 2012.

[31] A. Paliathanasis, M. Tsamparlis, and S. Basilakos, "Constraints and analytical solutions of $f(R)$ theories of gravity using Noether symmetries," Physical Review D, vol. 84, no. 12, Article ID 123514, 11 pages, 2011.

[32] N. H. Ibragimov, Elementary Lie Group Analysis and Ordinary Differential Equations, vol. 4 of Wiley Series in Mathematical Methods in Practice, John Wiley \& Sons, 1999.

[33] T. Feroze and A. H. Kara, "Group theoretic methods for approximate invariants and Lagrangians for some classes of $y^{\prime \prime}+\varepsilon F(t) y^{\prime}+y=f(y, y)$," International Journal of Non-Linear Mechanics, vol. 37, no. 2, pp. 275-280, 2002.

[34] P. J. Olver, Applications of Lie Groups to Differential Equations, vol. 107 of Graduate Texts in Mathematics, Springer, New York, NY, USA, 2nd edition, 1993. 

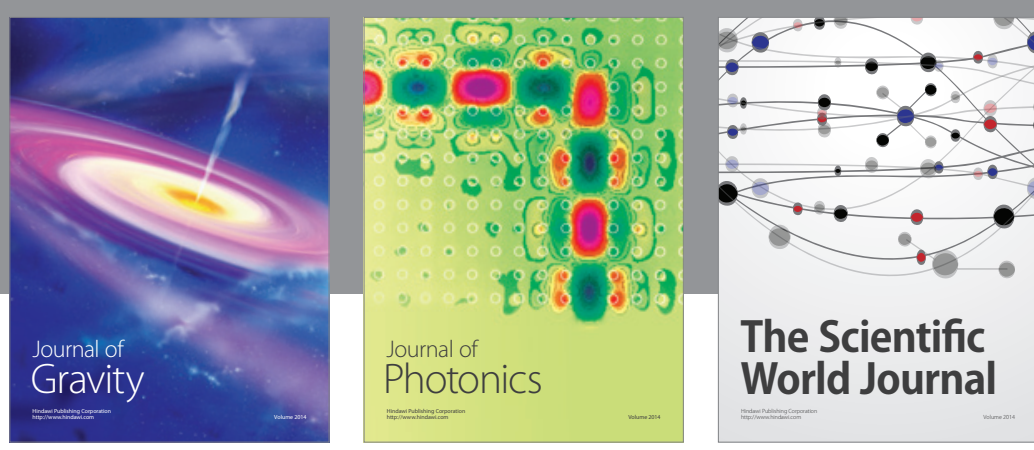

The Scientific World Journal
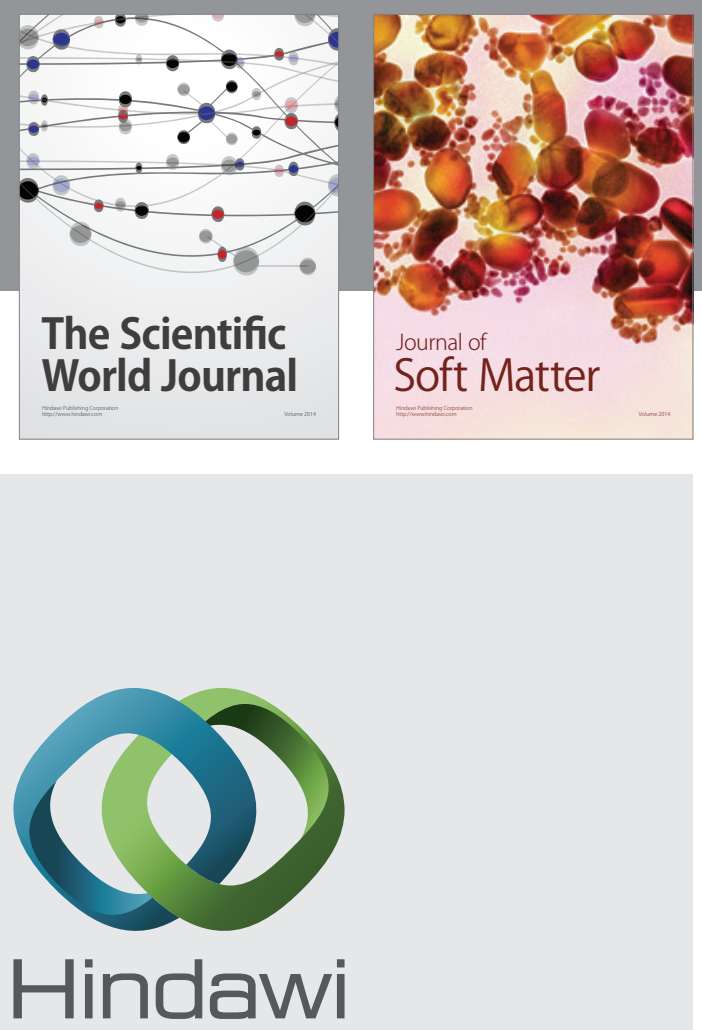

Submit your manuscripts at

http://www.hindawi.com

nternational Journal of

Statistical Mechanics
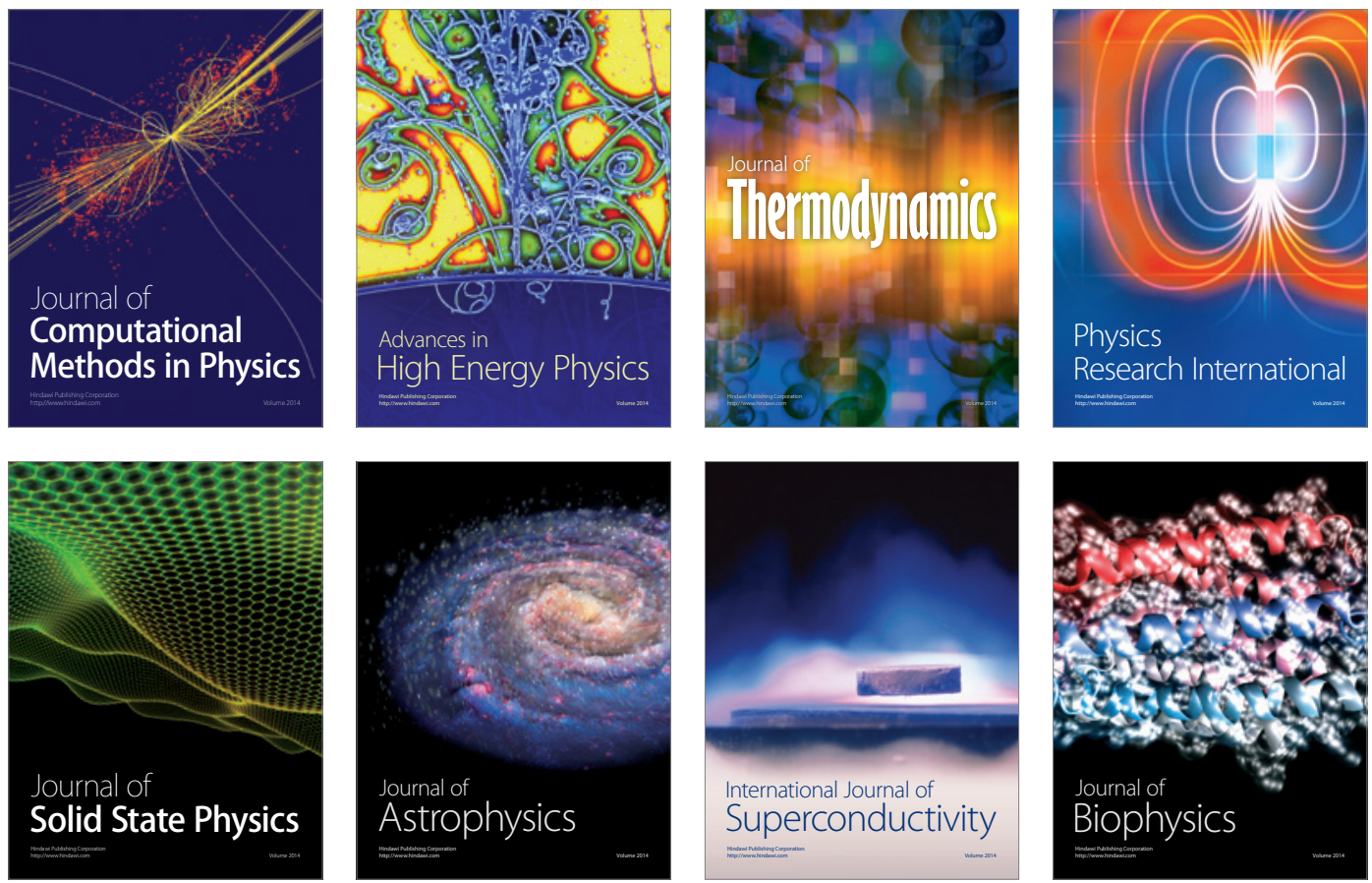
